\title{
Suicidal overdose of dolutegravir: A case report
}

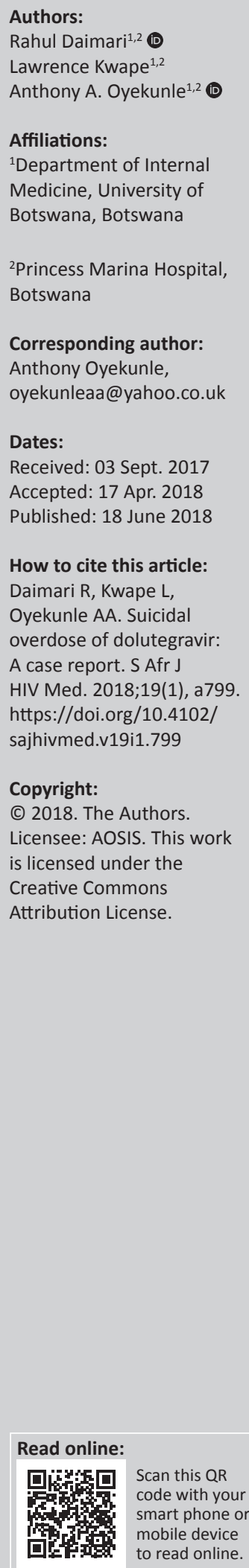

Dolutegravir (DTG) is the most recently introduced integrase inhibitor for the treatment of HIV infection and is preferred for its superior tolerability and efficacy in both new and pretreated patients, and infrequent drug interactions. Since January 2017, Botswana has adopted a 'treat-all' approach with a DTG-based antiretroviral (ARV) regimen as first-line treatment. We report a 29-year-old man with clinical stage 1 HIV infection who had been started on DTG, tenofovir and emtricitabine eight months prior, and who was admitted following a suicidal overdose of $1500 \mathrm{mg}$ of DTG. He reported only minor symptoms including vomiting, epigastric pain and dizziness; which promptly resolved following supportive treatment. On admission, full blood count, liver function tests and electrocardiography were unremarkable. However, there was a non-progressive increase in serum creatinine. After a month off ARVs, he was successfully restarted on antiretroviral therapy without any serious adverse effect.

\section{Background}

Integrase strand transfer inhibitors (InSTIs) are the newest class of antiretroviral (ARV) drugs approved for HIV treatment. Combination therapy based on InSTI is one of the alternative firstline ARV drugs recommended by the World Health Organization (WHO). ${ }^{1}$ Dolutegravir (DTG) is the most recent InSTI and is preferred for its superior tolerability and efficacy in both new and treatment-experienced patients and infrequent drug-drug interactions. ${ }^{2,3}$ Since June 2016, Botswana has adopted the 'universal test and treat' approach for HIV-infected persons, with a DTG-based regimen. ${ }^{4}$

\section{Ethical consideration}

For this case report, the authors obtained ethical approval from the Research and Ethics Committee (REC) of Princess Marina Hospital, Gaborone, and written informed consent from the patient. Ethics approval number is PMH 5/79(1-4-2018).

\section{Case}

We report a 29-year-old HIV-positive male truck driver, who was diagnosed with WHO clinical stage 1 HIV infection in May 2016, with a CD4+ count of 317 cells/ $\mu \mathrm{L}$, and was started on a triple regimen comprising DTG $50 \mathrm{mg}$ and tenofovir (TDF) $300 \mathrm{mg}$ and emtricitabine (FTC) $200 \mathrm{mg}$ in September 2016. The TDF/FTC were in a fixed dose combination, and DTG was provided as a separate pill. He achieved virologic suppression, with a viral load $<400$ copies / $\mathrm{mL}$ in December 2016. However, after an argument with his girlfriend, he ingested 30 tablets of DTG (1500 mg in total) with suicidal intention. He denied taking the other antiretroviral (ARV) drugs (TDF/FTC). He arrived at our hospital about $2 \mathrm{~h}$ after the incident and complained of epigastric pain, dizziness and vomiting. There was no prior history of depression or psychiatric illness. He drank alcohol only over weekends but denied smoking and substance abuse. On examination, he was conscious and well-oriented, with stable vital signs. Full blood count, liver and renal function tests were within reference ranges, although serum creatinine rose from $48 \mu \mathrm{mol} / \mathrm{L}$ (day 1) to $82 \mu \mathrm{mol} / \mathrm{L}$ on day 2 (Table 1). Serum level of DTG could not be done. Electrocardiography was normal, including a normal QTc. He was managed with intravenous fluid, metoclopramide and an antacid, and was off of his ARVs during his 3-day hospital stay. He was restarted on DTG and TDF/FTC after discharge, day 5 post-overdose. As per hospital protocol, he also had a psychological assessment and psychiatric consultation, which were unremarkable. CD4+ count on day 9 was 538 cells $/ \mu$ L. By day 28 follow-up he reported no serious adverse events and clinical assessment revealed no significant findings. Serum creatinine had also decreased to $72 \mu \mathrm{mol} / \mathrm{L}$. As at the last check-up 7 months after overdose, the CD4+ count had risen further to 828 cells $/ \mu \mathrm{L}$. 
TABLE 1: Changes in full blood count and serum chemistry during admission and follow-up.

\begin{tabular}{|c|c|c|c|}
\hline Complete blood count & Day 1 & Day 2 & Day 28 \\
\hline Haemoglobin (g/dL) & 13.7 & 13.9 & 14.7 \\
\hline Haematocrit (\%) & 38.7 & 41.0 & 42.2 \\
\hline $\mathrm{MCV}(\mathrm{fL})$ & 92.1 & 96.7 & 93.4 \\
\hline $\mathrm{MCH}(\mathrm{g} / \mathrm{dL})$ & 32.6 & 32.8 & 32.5 \\
\hline RDW (\%) & 12.4 & 13.2 & 12.7 \\
\hline White blood cells $\left(\times 10^{9} / L\right)$ & 6.55 & 4.60 & 7.61 \\
\hline Neutrophils (\%) & 44.5 & 37.7 & 59.7 \\
\hline Lymphocyte (\%) & 48.9 & 53.8 & 31.3 \\
\hline Monocyte (\%) & 5.2 & 4.8 & 5.1 \\
\hline Eosinophils (\%) & 1.2 & 3.3 & 3.5 \\
\hline Basophil (\%) & 0.2 & 0.4 & 0.3 \\
\hline Platelets $\left(\times 10^{9} / \mathrm{L}\right)$ & 176 & 212 & 220 \\
\hline \multicolumn{4}{|l|}{ Serum chemistry } \\
\hline Sodium (mmol/L) & 134 & 138 & 137 \\
\hline Potassium (mmol/L) & 4.2 & 5.1 & 4.2 \\
\hline Chloride (mmol/L) & 99.8 & 100.7 & 100.6 \\
\hline Urea (mmol/L) & 4.3 & 4.0 & 6.9 \\
\hline Creatinine $(\mu \mathrm{mol} / \mathrm{L})$ & 48 & 82 & 74 \\
\hline $\begin{array}{l}\text { Estimated creatinine } \\
\text { clearance }(\mathrm{mL} / \mathrm{min})\end{array}$ & 155 & 91 & 100 \\
\hline AST (U/L) & 31 & 30 & 30 \\
\hline ALT (U/L) & 28 & 27 & 17 \\
\hline
\end{tabular}

MCV, mean corpuscular volume; MCH, mean corpuscular haemoglobin; RDW, red cell distribution width; AST, aspartate aminotransferase; ALT, alanine aminotransferase.

\section{Discussion}

Integrase inhibitors are an essential addition to HIV treatment and act by blocking the incorporation of HIV DNA into the host genome, thus preventing replication. Drugs in this class include raltegravir, elvitegravir and DTG. Dolutegravir is taken once daily (50 mg for adults), has a good tolerability profile and superior virologic activity. It is primarily metabolised by the UDP glucuronosyltransferase (UGT1A1) pathway and excreted into bile. Hepatic cytochrome P4503A also contributes to DTG metabolism through oxidation by a minor pathway. ${ }^{5}$

Dolutegravir can thus be safely used in patients with significant renal dysfunction, without an expectation of renal impairment or dangerously high drug levels. Even though DTG is not known to have any direct effect on glomerular filtration rate, it impairs renal creatinine secretion through inhibition of the organic cation transporter-2 (OCT2) and may create an impression of deteriorating renal function. ${ }^{5,6}$ In addition, Guttierrez et al. mentioned in their review that there was a slight but predictable increase in serum creatinine concentration and a decrease in estimated creatinine clearance $(\mathrm{eCrCl})$ of about $15 \%$ among patients taking DTG at the pharmacologic dose and without an actual reduction in glomerular filtration rate. ${ }^{6}$ Our patient experienced a non-progressive but significant increase in baseline serum creatinine, which may be attributable to this effect. Hence, clinicians should be guided by other measures of renal function that are not known to be affected by DTG.

The occurrence of psychiatric symptoms including suicidal ideations in HIV-positive patients receiving DTG was reviewed across five randomised clinical trials by Fettiplace et al., and the authors concluded that similar to other ARV drugs, these symptoms were infrequently reported. ${ }^{7}$ Another review suggested that psychiatric symptoms may indeed be an InSTI class effect. Literature reports of DTG overdose are sparse, and thus its likely effects remain unclear. Lee $\mathrm{M}$ et al. reported a case of a suicidal overdose of a combination of DTG, TDF and FTC. ${ }^{8}$ In that case, the authors observed a non-progressive renal failure which was attributed to the effect of DTG, although TDF (a potentially nephrotoxic drug which the patient also used) may have contributed to the rise in serum creatinine. In our patient, where DTG alone was used, there was also a similar non-progressive elevation of serum creatinine. OCT2 inhibition by DTG can readily explain these effects in both cases, and this 'renal impairment' is typically reversible without any permanent harm. ${ }^{8}$

To our knowledge, there are no independent research publications regarding single overdose of DTG either in humans or animals. An FDA pre-approval review of nonclinical animal toxicity studies by the drug sponsor concluded that there were no significant adverse events after single-dose ingestion of $10 \mathrm{mg}-1500 \mathrm{mg}$ among rats, mice and monkeys. ${ }^{9}$ However, animals developed gastrointestinal inflammation and haemorrhage in similar studies following repeated short-term, subchronic and chronic daily dosing, at $10-1500 \mathrm{mg} / \mathrm{kg} /$ day. In addition, hepatotoxicity was noted in male monkeys, and this included hepatocellular necrosis and diffuse hepatocellular hypertrophy and vacuolation. ${ }^{9}$ These studies also established that the plasma levels achieved by these animals after a maximum of 1500 $\mathrm{mg} / \mathrm{kg} /$ day are approximately seven times what humans achieve after a $50 \mathrm{mg}$ once daily dose. If the pharmacokinetic relationship between the oral dose and plasma DTG levels remain linear at such doses, it will imply that humans can be expected to experience similar effects after just about $350 \mathrm{mg}$ once daily.

Therapeutic interventions are mostly supportive. Patients are unlikely to benefit from dialysis following an overdose because DTG is highly bound to plasma proteins. ${ }^{3}$ In this index case, we found no significant adverse effect after an overdose of $1500 \mathrm{mg}$. His minor symptoms disappeared with supportive treatment.

In conclusion, single-ingestion DTG overdose may be associated with minimal toxicity, as compared to repeated daily overdose, which may potentially result in gastrointestinal bleeding and hepatotoxicity. The apparent renal dysfunction noted in these cases is usually owing to OCT2 inhibition by DTG and is reversible. However, as experience is limited to the likely range of toxicities that may develop, physicians are advised to adopt measures that will prevent any form of overdosing using ARVs.

\section{Acknowledgements Competing interests}

The authors declare no potential conflict of interest. 


\section{Authors' contributions}

R.D. and A.A.O. conceived and designed the study, and conducted literature review. All authors were involved with the care of the patient, obtaining consent, data collection, writing and approval of the initial and final manuscript draft.

\section{References}

1. WHO. Consolidated guidelines on the use of antiretroviral drugs for treating and preventing HIV infection [homepage on the Internet]. World Health Organization 2016 [cited 2017 July 28]. http://www.who.int/hiv/pub/arv/arv-2016/en/

2. Despiégel $\mathrm{N}$, Anger $\mathrm{D}$, Martin $\mathrm{M}$, et al. Cost-effectiveness of dolutegravir in HIV-1 treatment-naive and treatment-experienced patients in Canada. Infect Dis Ther 2015;4(3):337-353. https://doi.org/10.1007/s40121-015-0071-0

3. Taha $H$, Das $A$, Das $S$. Clinical effectiveness of dolutegravir in the treatment of HIV/AIDS. Infect Drug Resist. 2015;8:339-352. https://doi.org/10.2147/IDR. S68396
4. Handbook of the Botswana 2016 integrated HIV clinical care guidelines [homepage on the Internet]. [cited 2017 Sept 24]. http://www.moh gov.bw/Publications/ Handbook_HIV_treatment_guidelines.pdf

5. Reese MJ, Savina PM, Generaux GT, et al. In vitro investigations into the roles of drug transporters and metabolizing enzymes in the disposition and drug interactions of dolutegravir, a HIV integrase inhibitor. Drug Metab Dispos. 2013; 41(2):353-361. https://doi.org/10.1124/dmd.112.048918

6. Gutiérrez F, Fulladosa X, Barril G, Domingo P. Renal tubular transporter-mediated interactions of HIV drugs: Implications for patient management. AIDS Rev. 16(4):199-212. [cited 2018 Feb 14]. http://www.ncbi.nlm.nih.gov/pubmed/

7. Fettiplace A, Stainsby C, Winston A, et al. Psychiatric symptoms in patients receiving Dolutegravir. J Acquir Immune Defic Syndr. 2017;74(4):423-431. https:// doi.org/10.1097/QAl.0000000000001269

8. Lee $\mathrm{M}$, Eyer F, Felgenhauer $\mathrm{N}$, Klinker HH, Spinner CD. Overdose of dolutegravir in combination with tenofovir disaproxil fumarate/emtricitabine in suicide attempt in a 21-year old patient. AIDS Res Ther. 2015;12:18. https://doi.org/10.1186/ s12981-015-0054-y

9. FDA. Center for drug evaluation and research [homepage on the Internet]. 2013 [cited 2018 Jan 17]. https://www.accessdata.fda.gov/drugsatfda_docs/nda/ 2013/2047900rig1s000PharmR.pdf 